\title{
Virtual REALITY OF AL-HARAM AL-MAKKaI EXPANSIONS USING 3DMAX AND ARCGIS SOFTWARE
}

\author{
Alanoud Salem ${ }^{1}$, Sara Musallam ${ }^{2}$, El-Shaimaa Nada $^{3}$ and Ahmed Ahmed ${ }^{4}$ \\ ${ }^{1,2}$ Department of Information System, Taibah University, Almadinah, KSA \\ ${ }^{3}$ Department of Computer Science, Taibah University, Almadinah, KSA \\ ${ }^{4}$ Department of Computer Enginnering, Taibah University, Almadinah, KSA
}

\begin{abstract}
Animated movies are excellent virtual environments for creating models in high quality. Animated movies can include 3D models, sounds and lights effects, and detailed maps. In this paper, a virtual reality movie is applied to Al-Haram Expansion stages including the future stage of expansion. 3DMAX program is used to rich the maximum benefits of using 3D modeling. Maps with details are built by using ARCGIS program in order to understand the real difference between the three different expansions stages clearly and effectively. A novel technique is presented in order to insert $2 D$ maps and other details in the $3 D$ model built by $3 D M A X$.
\end{abstract}

The animated movie includes introduction, three basic expansion stages, maps, and conclusion. It is in Arabic with translation to English and sign language is also included. Accurate and documented information is applied in AL-Haram expansion movie that helps people to know about one of the most radical expansions that is occurred in the world.

\section{KEYWORDS}

VRML, VR, 3DMAX, ARCGIS, modelling, 3D models

\section{INTRODUCTION}

Virtual Reality (VR) is a popular system for simulating reality using computer technology that makes the user indulged in the environment. It makes the world to be real enough, using enormous computing power.

Our work presents a 3D modeling show on the stages of the expansion of Al-Haram Mosque in Mecca. The movie contains the different stages of the past, the present and the future and the changes that have been taken place on each stage. Mapping of the expansion stages is also included. 3DMAX and ARCGIS software are chosen in order to create a high quality 3D model with accurate map of each stage.

There is a continuous increase over time to expand the Al-Haram Mosque in Mecca in Saudia Arabia. For the best of our knowledge, there is no such accurate and documented information about the stages of the expansions of Al-Haram Mosque that has been considered in any previous work.

In this work, Displaying of expansions will include the details of three stages in 7 minutes of $3 \mathrm{~d}$ model. To create a high quality model, 3DMAX modeling software is used with sound, light features. Static maps are also included to clarify the expansion of each stage using state of art ARCGIS software. The movie will be in Arabic language and will also be translated to English. 
Our goal is to reduce the time of modeling by using 3DMAX program, that provide visual environment rather than wasting time in coding, to achieve the required interaction between the elements that's in virtual environment by the correct use of tools (sound, light for example) that come with the modeling software, to give the right perception for the events that occur in the AlHaram by present the scene as virtual environment that simulate the reality in mutable site especially official events like al-hajj, and to clarify the need of the expansion, and to improve the quality of the models made by VRML by merging between $3 \mathrm{~d}$ modeling of the environment of study and static maps that show the changing details of the this environment.

A novel approach of inserting 2D maps designed by ARCGIS into 3DMAX program, this is done to convert these maps into 3D and to add more features to them such as sound effects, color and reduce errors of small details of the maps.

In next section, a literature review is introduced. In section 3, virtual reality of Al-Haram New Expansion is presented. In section 4, an analysis of online questionnaire is applied. The creating models and proposed technique is presented in section 5. The results of the implementation of the movie with 3DMAX and ARCGIS are in section 7. Testing of the movie is in section 7. The conclusion and the future work is in section 8 .

\section{LITERATURE REVIEW}

The application of VR causes the classical architecture used for protection and a demonstration to be more scientific, effective and appropriative. It also responds to a request from the property and the current information age. Its goal is to use enormous computing power and chart an exaggeration computer capacity, to make the assumption that the world seems real enough. In this section a description of some related work using VR is presented.

In [1], it presents Crowd Simulation in Emergency Aircraft Evacuation using Virtual Reality CGAMES. VR can be used to produce a realistic environment involving multiple sensory channels VR crowd simulation can be used by airlines to simulate emergency scenarios without the use of live actors. But in this work, there are some considerations such as: The whole system is complex to implement. It didn't simulate each case of emergency that might happen. It didn't consider the external environment enough that can effect in the plan. The programs used in modelling this environment are 3DMAX, Maya program and Virtual Reality Modelling Language (VRML) Virtools.

In [2], it shows the Virtual Reconstruction of FouGuang Temple Based on Virtual Reality. In this work, the history of the humanities and browsing the heritance physical geography roaming is presented. Protection, introduction of humanities history, geography and physical side can play very vital role in the international virtual reality technology. The limitations of this work are: Control buttons on the screen are not provided by the VRML browser and some building components have complex geometric models this leads to some difficulties in modelling all components. It is hard for the user to observe all the details carefully because details of modelling are complex to be modelled. The program used for presenting this $3 \mathrm{D}$ environment is $3 \mathrm{DMAX}$.

In [3], it presents Relief Mapping on Facade of Sino Portuguese Architecture in Virtual Reality it presents a mapping technique to reduce the number of polygonal built in model. Virtual reality is applied to a door, wall and house. Graphic card is used to apply great shadows and lighting in image. Implementation of VR for a model is done to present a Walk through the environment to give information in real time. The program used for building the 3Dmodel is 3DMAX. 
The work in [4] aims to investigate the role of Virtual Reality and three-dimensional (3D) computer modelling on learning and teaching in a school of the environment. In order to achieve this aim a number of academic experiences were analyzed to explore the applicability and viability of 3D computer modelling and VR into the modelled environment areas. Although 2D representations have been greatly accepted for obtaining professions and education environment model, 3D computer representations and VR is still required. The general characteristics of this work are: Offering an approach on how three-dimensional computer modelling and virtual reality may be integrated into built environment teaching. A strategic, systematic approach was adopted to raise awareness of VR technologies in the school. A total of 11 semi-structured interviews were conducted with key participants involved in the integration of 3D technologies into the built environment curriculum. Each interview lasted one hour and was audio-taped to facilitate data analysis. After careful consideration of location and needs of users, the facility was centrally situated in the heart of the school, to allow easy access and to promote this technology to students, staff and visitors. Allowing staff and students to view designs in stereoscopic format, from multiple viewpoints, and navigate through space in real time. The aim from the outset was to foster VR applications across all disciplines within the School and to encourage collaboration with local practices and other researchers. The programs used for building this school environment are 3DMAX and VR4MAX.

In [5], it created the 3D model of Multipath by loading models in other software or programming by using Arc Object, because it cannot be directly created by ARCGIS. The methods used to create virtual campus are studied based on 3D modelling, database and image processing, such as using 3DMAX to create 3D model and putting on the texture image, and by ARCGIS special analysis and C\# redeveloping specific function. It provides more credibility guarantee for the campus planning and sustainable development. The model of virtual campus compiles with image, text, sound, video to create a vivid 3D campus, which is basis and platform of the digital campus to allow users to access virtual environment. In this work, Data digitization is done as in the following steps:

- Importing the base maps: firstly import into ArcMap the campus planning maps.

- Raster and rectify: because of lacking their geographic coordinates the existing planning map data need to rectify.

- Creating the elements layers: it is used create appropriate layers file in ArcCatalog, base on the ruler of graphics and objects, combined with the practical needs of the virtual campus.

- Vectorization on screen: Adding layer file that created in the ArcCatalog to ArcMap, and activating the ArcScan expansion toolbar.

The programs used in this work are 3DMAX, Arc GIS and Photoshop and the programming languages are C\# and SQL.

In [6], it deals with Suzhou University of Science and Technology as an example, designed a realtime virtual campus roaming system. It discussed the three-dimensional modelling of buildings, gates, roads, bridges, surrounding lawns, trees and other objects on campus, and implemented the data change between 3DMAX and VRML Pad. At the same time, it used a model with kinds of texture maps, multi-resolution hierarchy, and achieved the scene using DEF/USE ways of VRML language. This work focuses on some details such as a variety of scene building, scene model and mapping methods, code conversion, optimization techniques, interactive programming based on the triggers, cruise, controlling viewpoints and communication between JAVA and scene. 


\section{Virtual Reality OF Al-HaRam NeW ExPansion}

Although the New expansion of Al-Haram is a great event especially for Muslims, we found no scientific research on the details of the new expansion. There are many videos which show the steps of the expansion of Al-Haram but most of them have a lack of documentation and clarification and even the information cannot be completely trusted.

It is worth to mention here that there are some videos on Al-Haram expansion which has appeared recently by Al-Haram ministry of foreign affairs.

This documentary show in [7] presents the project of expansion space of Almtaf during nine minutes nearly and by Arabic language.

The advantages of this show are:

- The time period to present the project is good.

- The film is supported by date.

- The problem is well defined.

The disadvantages of this show are:

- The film doesn't have more than one language.

- The film contains duplicate scenes.

- $\quad$ Like most other films is talking about some part of expansion.

- The film doesn't contain any illustrative maps.

In our work, 3DMAX software is used to make movie about the stages of the expansions of AlHaram mosque in Mecca with providing accurate and documented information in clear and simple way. The movie will take 7-minutes and includes introduction, three stages and conclusion. The three stages are King Fahd Expansion that is happened in the past and King Abdullah Expansion in the current period and king Salman Expansion in the future. We selected these stages because these stages made radical changes in Al-Haram.

The movie will be in 3D modelling for all objects in order to achieve a very understandable movie, virtual reality is applied because of its advantages in the speed, ease and information accessibility for the recipient with the best representation.

In addition to the movie, maps will be added at the end of the movie by using ARCGIS software. The maps will be for the three stages which describes the expansion in the form of illustrations and detailed maps. We add effects such as sound, translation to achieve the required interaction between audience and the movie. The main advantages of the presented work

- Conveying the information in a simple, clear in the shortest possible time.

- Presenting accurate and trusted information as the information comes from an accredited Side (Al-Haram development staff).

- Adding a translation of the video to benefit the most people, especially non-native speakers of Arabic.

There are some limitations of our work such as using only two languages (Arabic, English) Multilanguages and sign languages movie will be our next step. 


\section{ANALYSIS OF APPLIED QUESTIONNAIRE}

At the beginning, an online questionnaire has been done to determine the user requirement that will be used in making the movie. A survey contains three groups: Group number one includes general information (Name, Age, Gender, Education). We asked about the age to know which age group have enough information, the Gender will tell us which part of the society have more interest in having information on the expansions, and the benefit of education question is to clarify how the people are effected with their level of Education and how this will be impact in their interest.

The description of Results from group number one are presented in Figure 1, the results show that $30 \%$ of the people who did the survey were female and $25 \%$ of the people were male , and most of them their age were above 30 and our survey clarifies that they are interesting in AlHaram expansion information.

Group number two is about Al-Harm Expansion, it discusses the information that people have , and their interests about getting information about Al-Haram expansion, how they prefer to get information about Al-Haram Expansion, how they want us to model the movie (3D,map,photos), what is the appropriate time for them to present the movie, and which language is more suitable for them.

The description of Results from group number two are presented in Figure 2 presents that $52 \%$ of people want to get information about Al-Haram expansion in form of movie and $43 \%$ of them want to that include maps, photos, and they want it in 3D form, and $26 \%$ of people prefer to present data based on King who has been during his reign and the time of its occurrence.

It also shows that $36 \%$ of people want the information of Al-Haram expansion to be visible and heard and $43 \%$ of them prefer the time to be around 10 minutes and $32 \%$ want the language of the movie to be in Arabic.

In Group number three we asked them about the future work they want it to be available in our movie (sign language, sound effects, detailed map). The description of Results from group number three are presented in Figure 3, it shows that $44 \%$ of people prefer to add sign language and $51 \%$ of them want to place sound effects and $47 \%$ want to see maps in the movie.

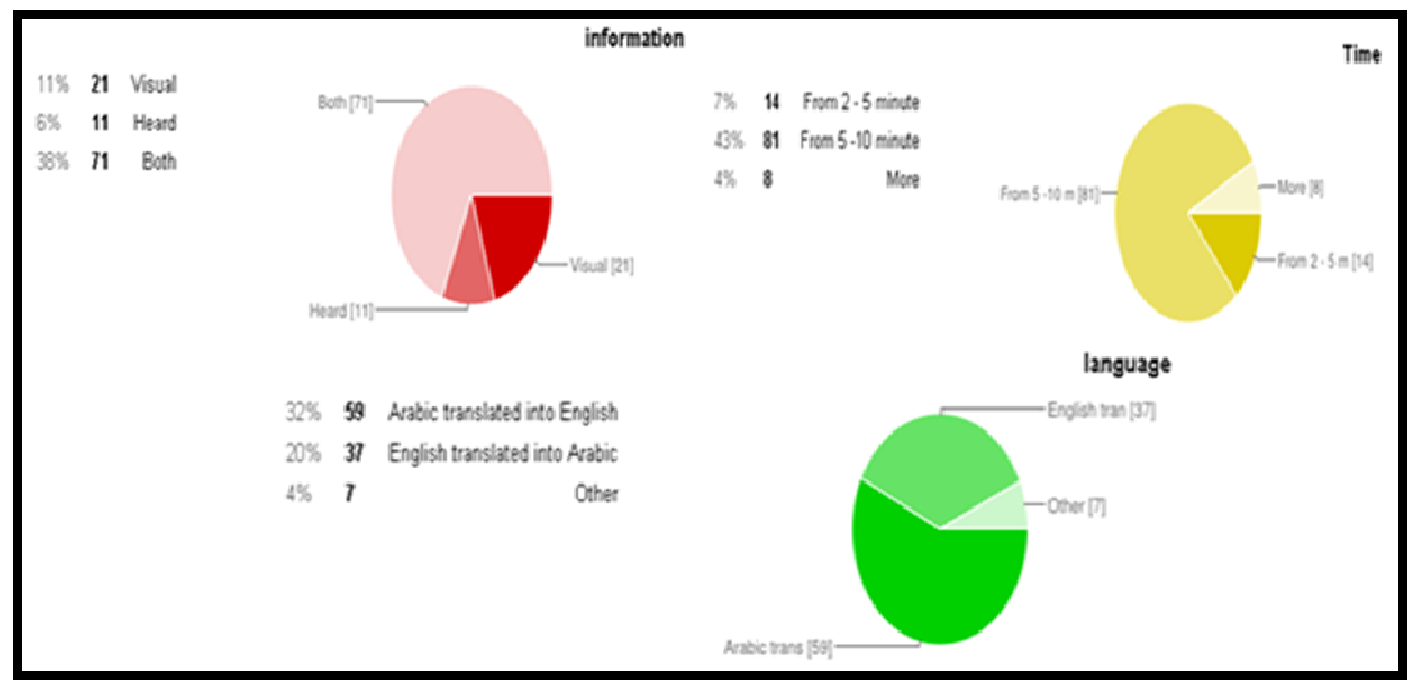

Figure1. Results from group number one 


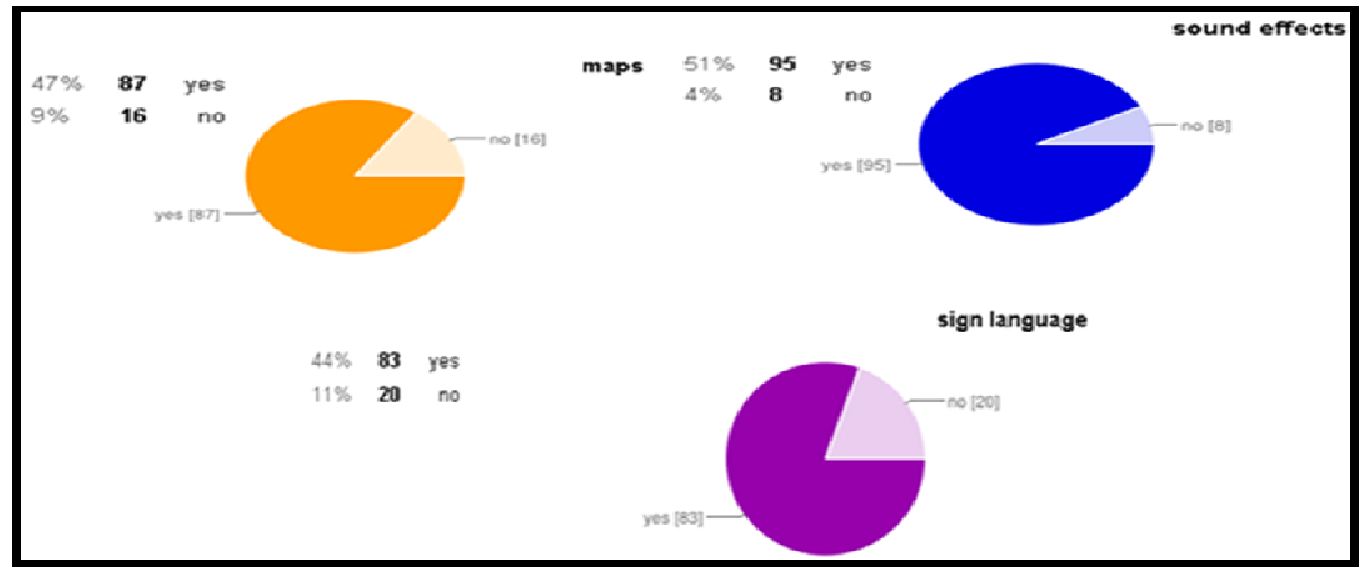

Figure 2. Results from group number two

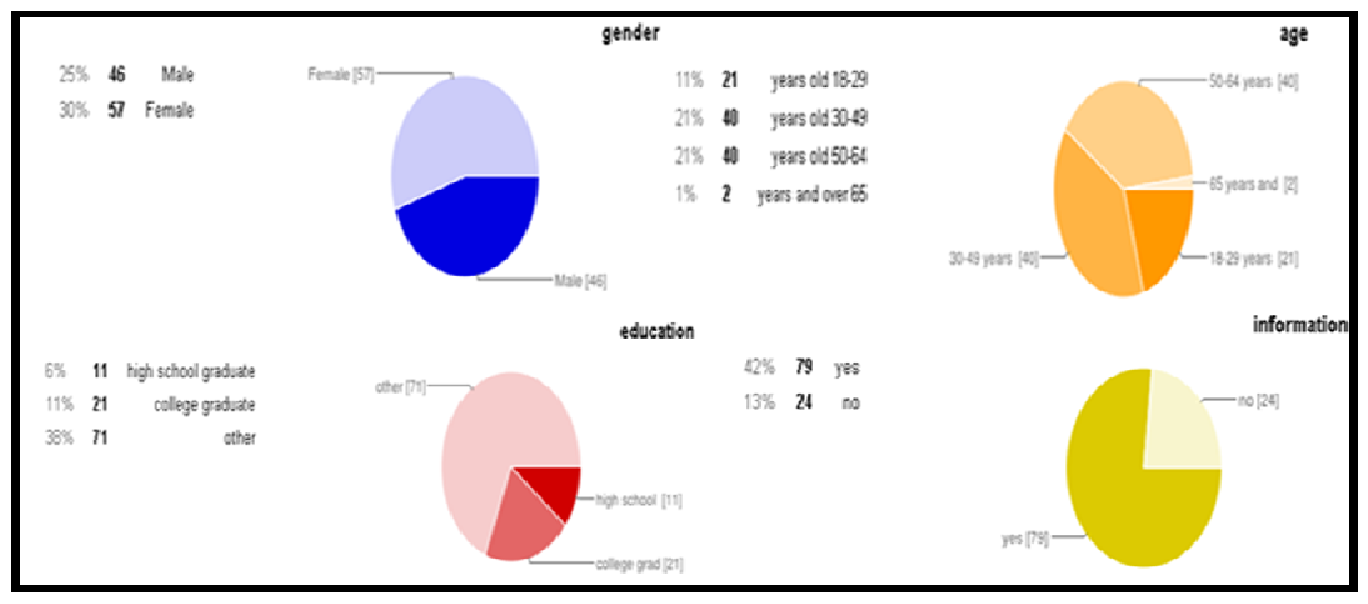

Figure 3. Results from group number three

\section{CREATING MODELS FOR THE PROPOSED TECHNIQUe}

The description of systems architecture of Al-Haram movie is presented in Figure 4, it contains:

- Introduction: the movie of al Haram new expansion starts with projection to Makkah the voice will tell general information about Makkah and special place of Makkah for muslims.

- $\quad$ Stage 1: It starts with 3D image of king Fahd (the expansion occur in his time) and will display information about the first stage (in the past) of expansion.

- Stage 2: It starts with 3D image of king Abdullah (the expansion occur in his time) and will display information about the second stage (in the present) of expansion.

- Stage 3: It starts with 3D image of king Abdullah and king Salman (the expansion occurs in his time) and will display information about the third (future) stage of expansion.

- Maps: They will display descriptive maps of the three stages.

- Conclusion: the movie will end with the benefits of this expansion in Muslims life and display how it's suited with the increasing number of Muslims. 
The proposed technique in this paper is based on inserting Alharam Expansions maps designed by ARCGIS into 3DMAX program to make a complete view of the movie.

The created maps should be converted into3D before importing it, and then modification of the $3 \mathrm{D}$ maps is required. Because the small error is difficult to be found and the errors in the original images are hard to be modified, we smooth the maps that have been imported from the ARCGIS. This will increase the accuracy.

In 3DMAX, the models of the buildings are designed and the effects of the buildings are rendered. An example of Al- Kahba is shown in figure 5.

In this work the virtual buildings construction is an important part. Two methods are used in order to model and construct the virtual environment

- First: construction of virtual environment using 3DMAX.

- Second: convert the 3D model to VRML code.

The advantage of this method is that it is easy for constructing complex building but the code will become long and to solve this problem we do not choose a Normal's option, Where Normal's option is used for exporting the geometry of 3DMAX and makes VRML files very larger.

Using 3DMAX to create models of visual environment it taken shorten time of coding and debugging but only when we have a good model design that was created before.

Hybrid between the methods which enable back and forth switching from visually oriented tools to line editors and great high quality of model.

An example of one of created maps using ARCGIS program is presented in figure 6.

The figure shows how to create the map form the projection step to the final step to present a complete map. Figure 7 shows the final map of first stage.

There are many considerations while building Models such as:

- Data pretreatment: information and required data about the expansion is obtained from $\mathrm{Al}$ Haram development staff.

- Modeling of building: Complicated features, such as building details and Minarets are very difficult to create. We use AutoCAD with 3DMAX software.

- Optimizing the virtual system: The virtual system must keep proper frame frequency. Some optimizing approaches have been applied in the scene.

- 3D manipulations: 3D manipulations including zoom-out, zoom-in, group movement, shadows, and multi-angle shots have been realized.

- Adapting Virtual Reality for Design: VR is applied to provide several phases of improving the design of the area.

- Creating the environment: Creating the environment with the small details such as shadows and clouds is important to make the scene look more real, and to get the user attention.

- Import audio: to make the movie more attractive, importing audio is require, the audio will be played from the begging of the movie to explain the scenes and to give information to the audience.

- Layout design: The VR models permit 3D visualization and fly through animation to represent environmental quality, based on quantifiable image parameters, and assist the understanding of the complex. Without a VR system, the details could be evaluated only after completion of construction. In the design stage, it gives the ability to visualize potential modifications to the space. 
International Journal of Database Management Systems ( IJDMS ) Vol.7, No.5, October 2015

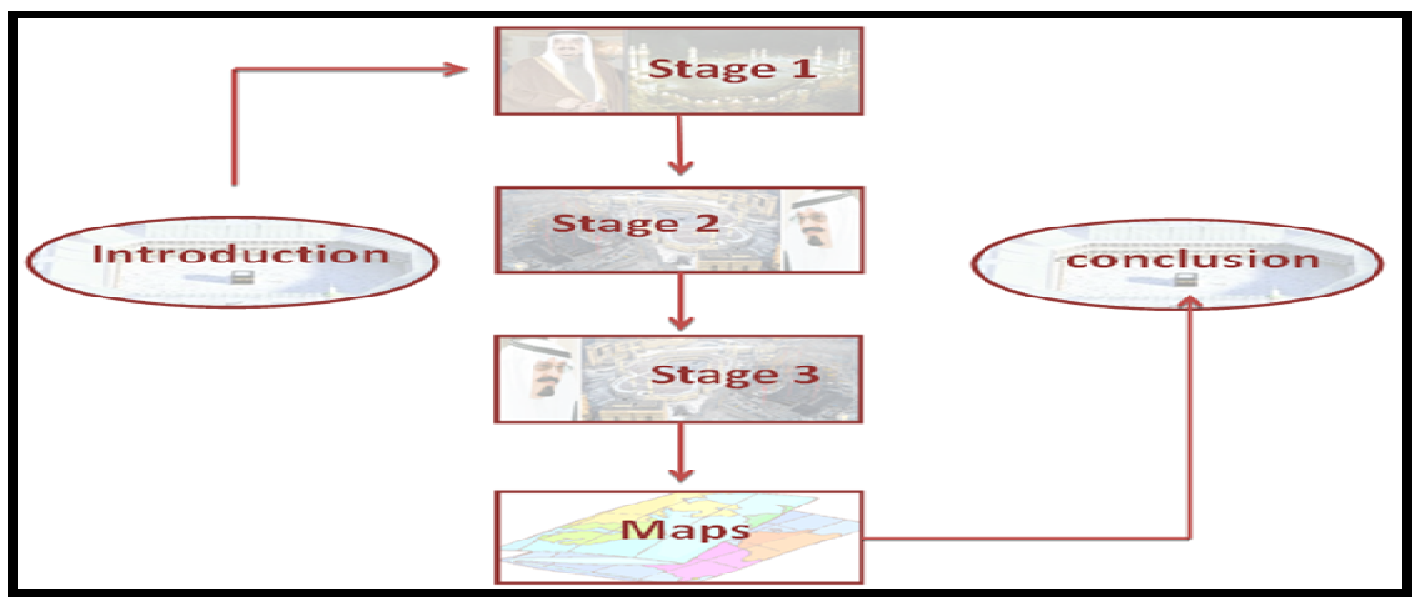

Figure 4. System Architecture of Al-Haram Movie

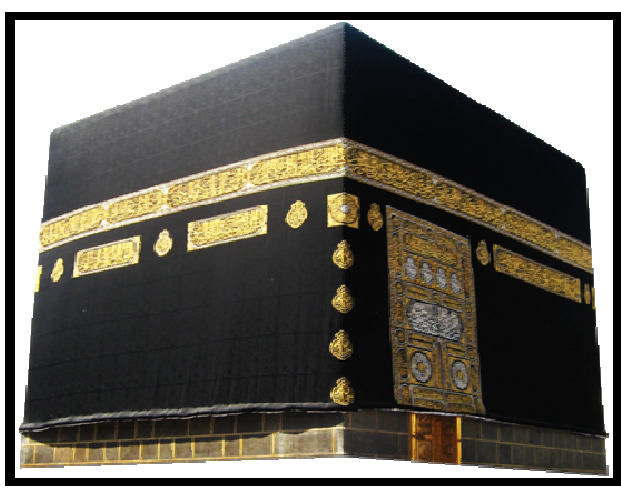

The real image

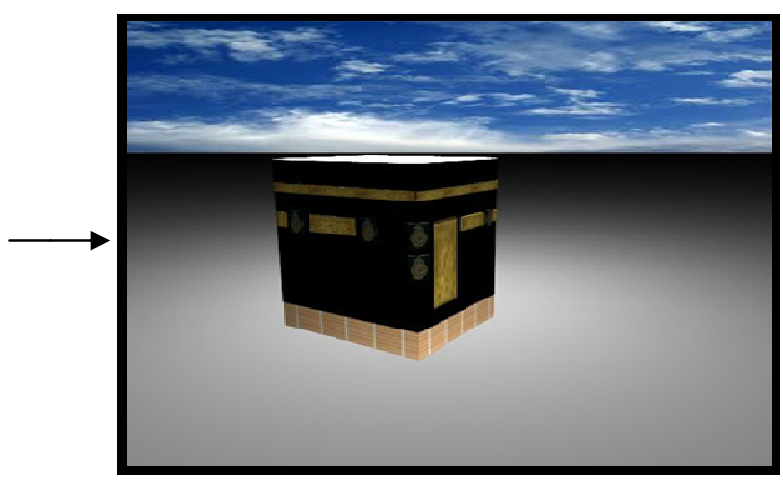

Implementation in 3DMAX

Figure 5. Creating environment that looks like the real image

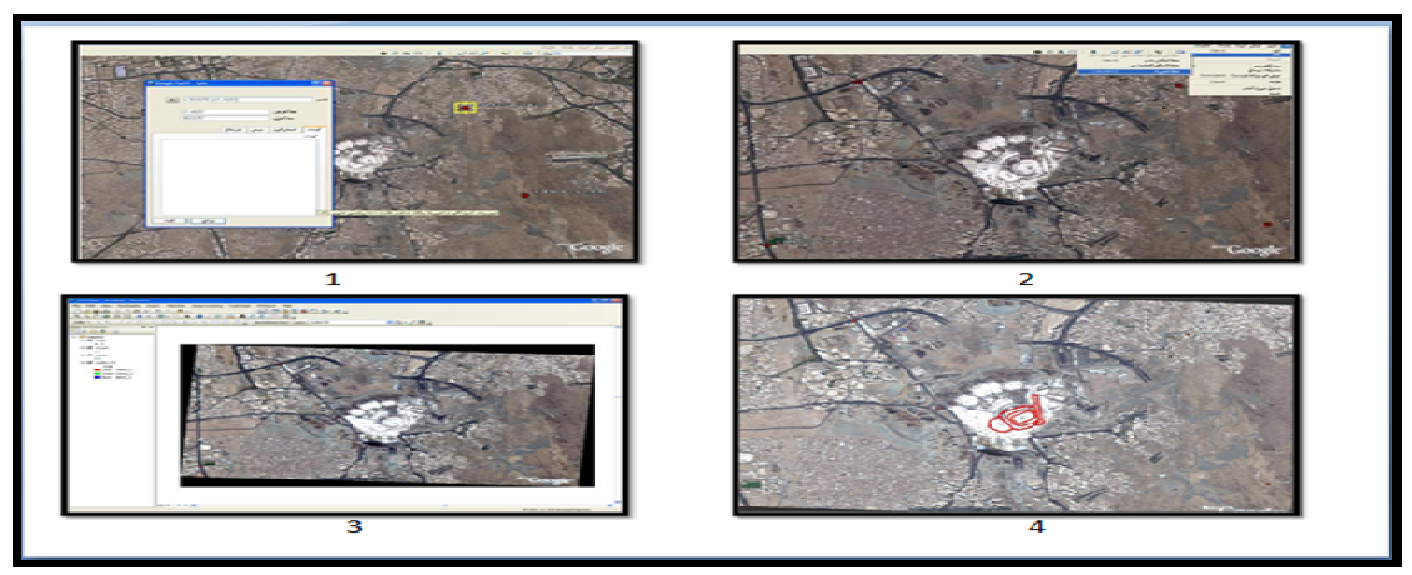

Figure 6. Designed maps using ARCGIS 


\section{RESULTS}

The final scene of all added object together, material and directed (add ligth ,cloud ) is adeded as in figure 8. The Expected extension of Al-Haram is presented in figure 9, it shows that this expansion will includes the Clock Tower. Acloser look is presented in figure 10. The final maps of the three extension that have been resulted is shown in figure 11.

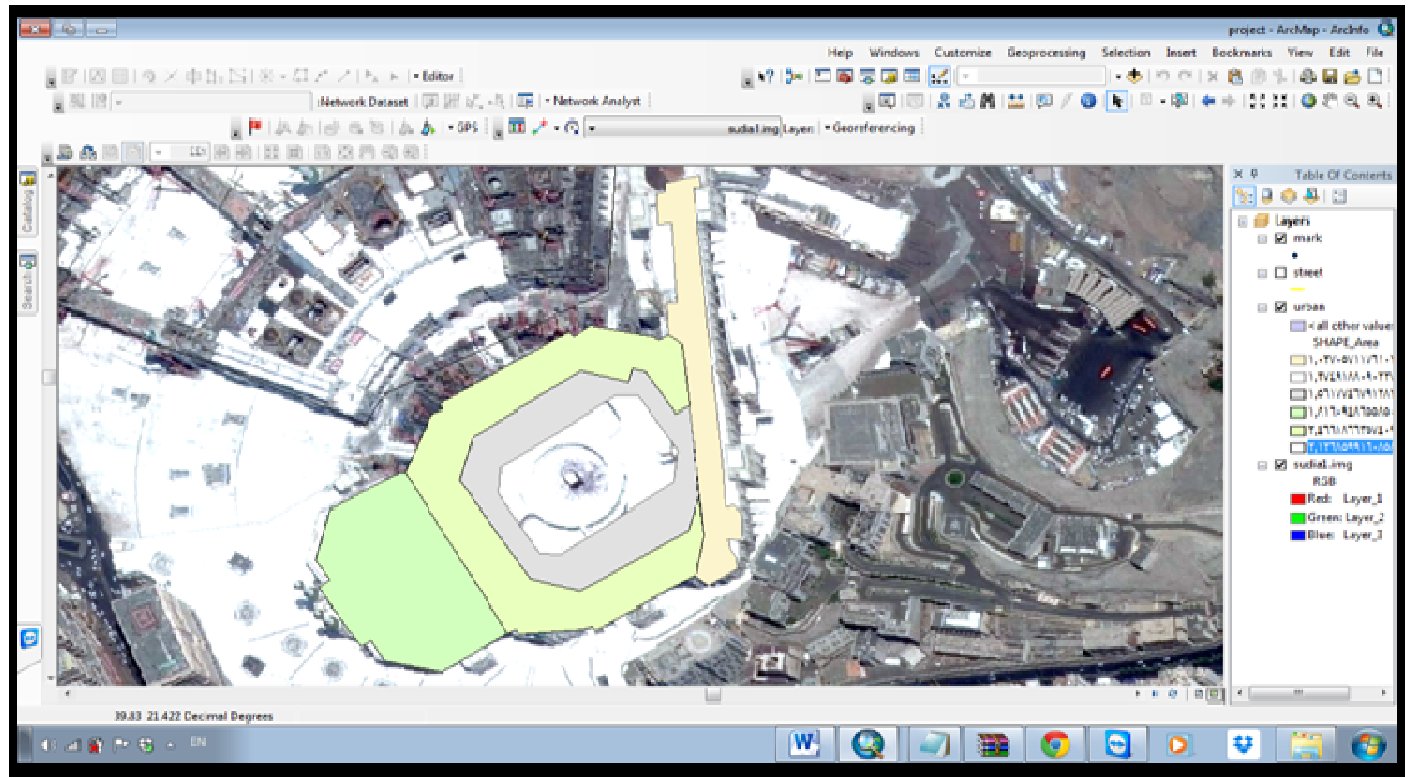

Figure 7. The final map of first stage

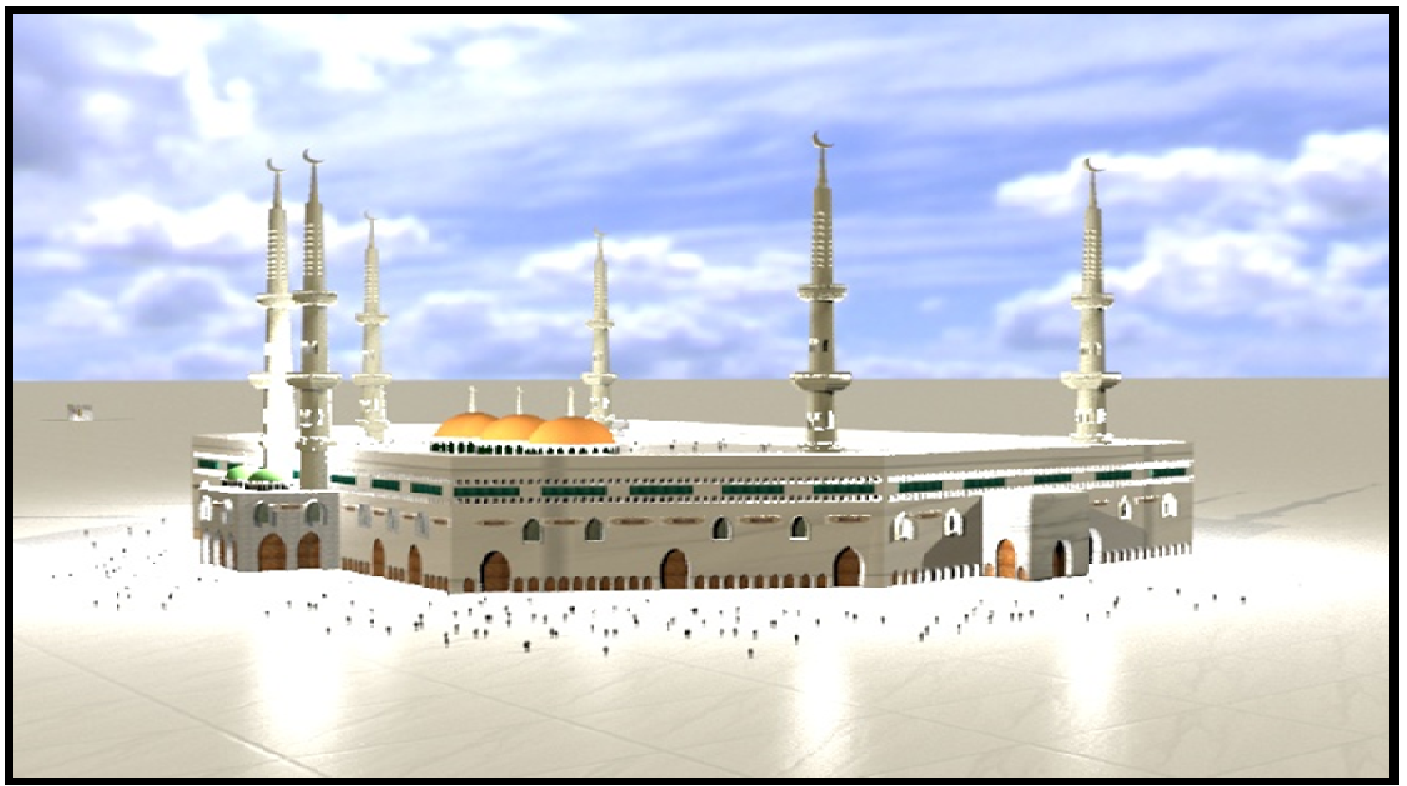

Figure 8 Final scene 


\section{TESTING}

An appropriate test of our project is to perform a survey to get feedback from people; we obtain the information from 100 students who study Geographical Information System course. The survey contained 5 questions about our movie.

$78 \%$ approves on the clarity of the sounds, $91 \%$ saws that the quality of the maps and images are very good, $62 \%$ said the movie sounds like real, $62 \%$ said that the movie is interesting, and 58\% saw that the movie's information is sufficient.

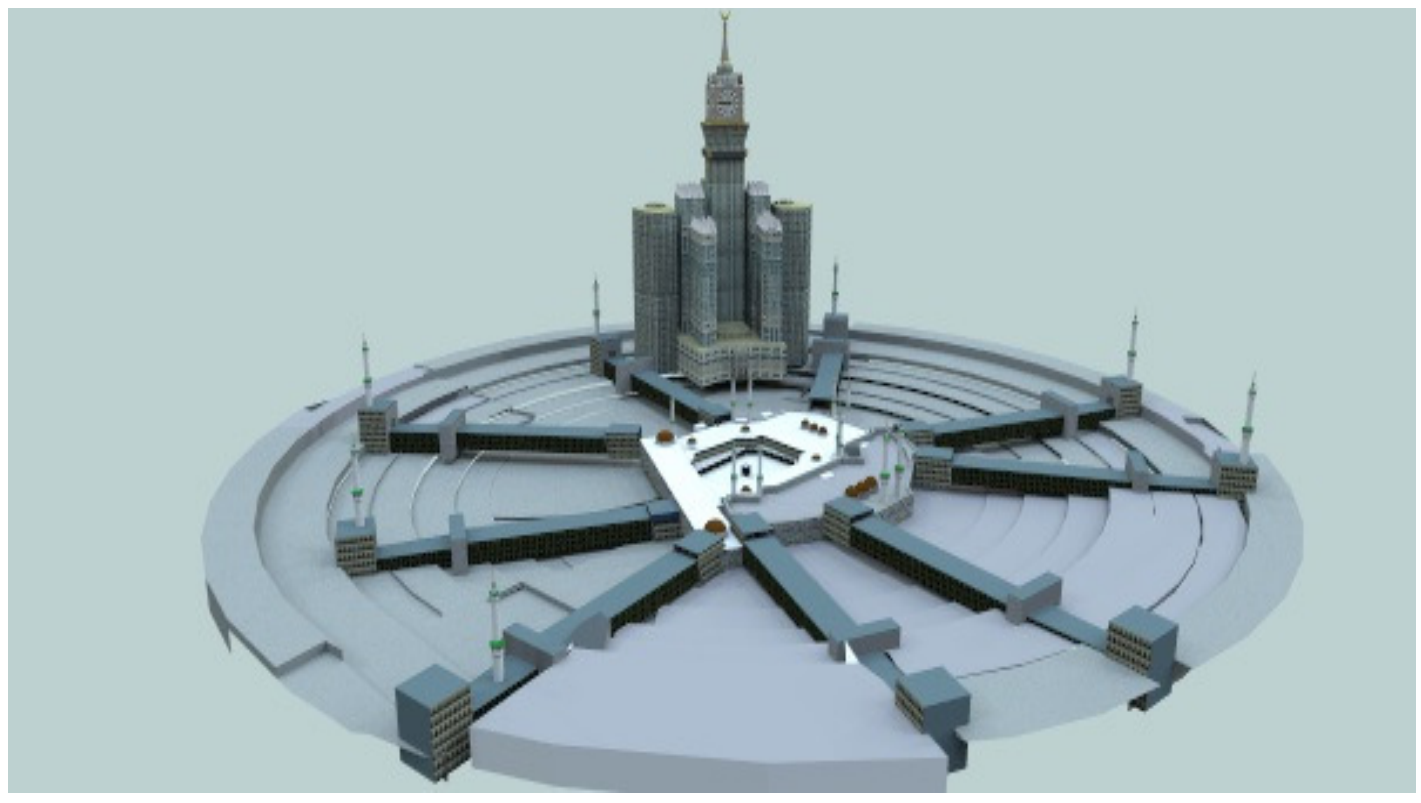

Figure 9 Expected Extension includes the Clock Tower

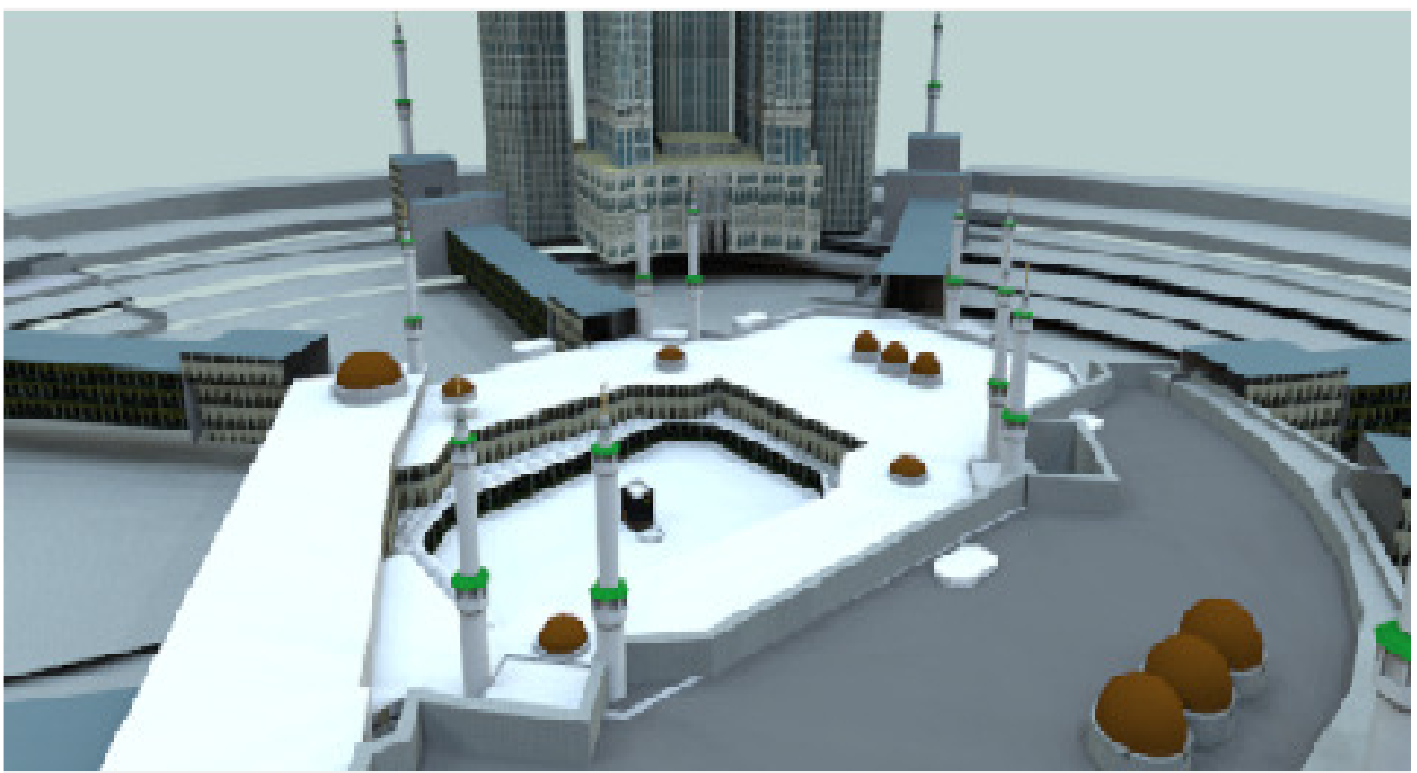

Figure 10 A closer look of Expected Extension 


\section{CONCLuSion}

In this paper, VR of Al-Haram Expansion Movie is presented. The last recent three stages of AlHaram expansion are shown with 3D maps of every stage. A novel technique of converting the 2D maps designed by ARCGIS program into 3D maps designed by 3DMAX program is presented. Many features are added in the movie such as sound, audio, translation. The movie is presented in Arabic and is translated in English. Sign language is added.

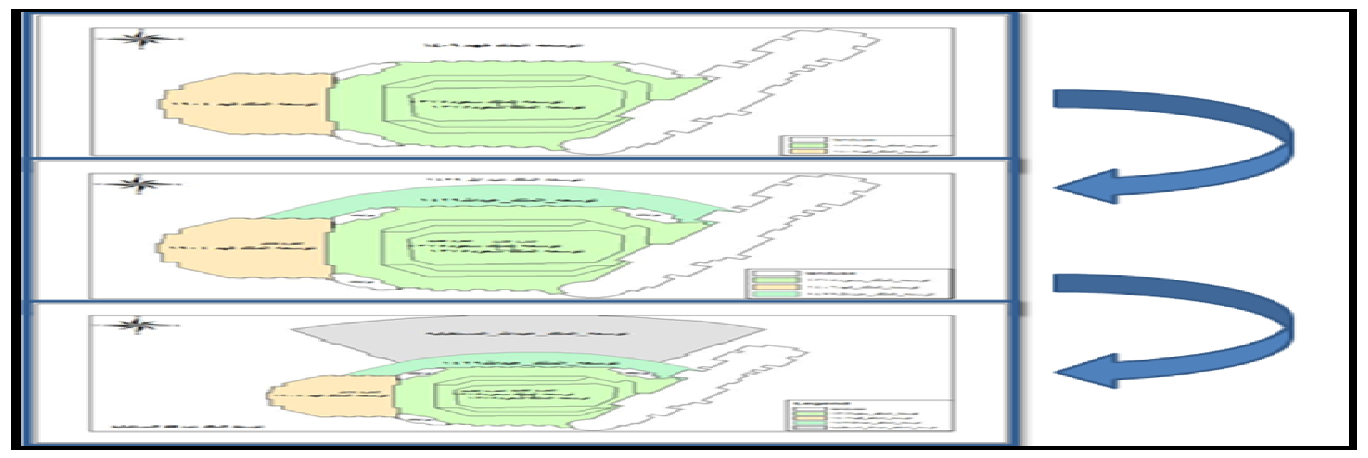

Figure 11 The creating map by ARCGIS

\section{REFERENCES}

[1] Sharad Sharma, Stephen Otunba, and Jingxin Han .(2011) "Crowd Simulation in Emergency Aircraft Evacuation using Virtual Reality" . USA: Department of Computer Science.

[2] Lu Shi Zhu. (2008) "Virtual Reconstruction of FouGuang Temple Based on Virtual Reality" . Nanchang: IEEE.

[3] Kalarat, K. (2014) "Relief Mapping on Facade of Sino Portuguese Architecture in Virtual Reality". Fourth Bangkok : IEEE.

[4] Margaret, Emine , (2007) International Conference on Construction Applications of Virtual Reality. UK: Northumbria.

[5] Shiliang Zhanga, Xiaobinhuangb , (2012) "Development of virtual Campus System Based on ARCGIS. Department of Information and Engineering Ningde Normal University Ningde, Fujian", Province, China: by Elsevier Ltd. Selection and/or peer-review under responsibility of ICMPBE International Committee.

[6] Zhao Mei-Hong.(2012) "Design and Implementation of Modeling Virtual Campus", Vol. 1, No.4. North University of China: IACSIT.

[7] Information available from their website (http://www.mofa.gov.sa/Pages/Default.aspx , 2005).

\section{AUTHORS}

Ahmed Abdul-Monem Ahmed received his MS and PhD degrees from the University of MissouriColumbia (currently University of Missouri). He is currently working as an assistant professor with Zagazig University, Egypt and Taibah University, Saudi Arabia. His research interests include wireless networks, virtual reality, and distributed systems.

El-Shaimaa Nada El-Shaimaa received his MS and PhD degrees from the University of Zagazig- Egypt. She is currently working as an assistant professor with Zagazig University, Egypt and a lecturer at Taibah University, Saudi Arabia. Her research interests include Intelligent System, virtual reality, and Control systems.

Alanoud Salem Alanoud is a student in Information System Department at Taibah University, Saudia Arabia. Her research interests include Virtual Reality, Geographic Information System.

Sara Musallam Sara is a student in Information System Department at Taibah University, Saudia Arabia. Her research interests include Virtual Reality, Geographic Information System. 